\title{
Evaluation of Ultrasonography in the Diagnosis of Acute Appendicitis with Histopathology as Gold Standard
}

Ahmed N. Manea; ${ }^{2}$ Tawfeeq J. Mohammad; ${ }^{3}$ Sarmad j. Shehatha;

\section{ABSTRACT}

Background: Acute appendicitis is the most common surgical abdominal emergency with a life time prevalence of 1 to 7 individuals. Because the clinical diagnosis of acute appendicitis remains a challenge to surgeons, so different aids were introduced like different scoring systems, computer aided programs, ultrasonography, computerized tomography, Magnetic resonance imaging, Gastrointestinal tract contrast studies and laparoscopy to improve the diagnostic accuracy.

Objective: To evaluate ultrasound in the diagnosis of acute appendicitis in those patients clinically diagnosed with histopathology as gold standard.

Methods: A cross sectional study carried in Alkindy Teaching Hospital through one year duration from $1^{\text {st }}$ of may2015 to $1^{\text {st }}$ of May 2016. All included patients were subjected to ultrasonographic examination to assess the vermiform appendix with a graded compression technique. The Ultrasonography findings were recorded as positive and negative for acute appendicitis.

All the appendices removed from the study patients were sent for histopathological study. Statistical analysis done using (SPSS) version 21, Chi-sequare test used for categorical variables and t-test was used to compare between two means. Level of significance (P value) set at $\leq 0.05$.

Results: A total of 215 patients with suspected appendicitis, males $112(52.09 \%)$ and females $103(47.9 \%)$ were included in present study. The validity results of ultrasound in comparison with histopathology findings were as following; accuracy $86.5 \%$, sensitivity $86.5 \%$, specificity $86.6 \%$, positive predictive value $99.8 \%$ and negative predictive value $32.5 \%$.

Conclusion: The ultrasonography has a good accuracy, sensitivity and specificity in diagnosing acute appendicitis.

Key words: Acute appendicitis, ultrasound, histopathology

1 Family Medicine Specialist, MBChB, HDF.M Corresponding author

E mail; drtwfkjm66@yahoo.com

Consultant Surgeon, MB.Ch. BFIBMS

Physician, MBChB, DGS

\section{INTRODUCTION}

7 vermiform appendix is, a worm shaped tubular structure projecting from the blind end of the caecum ${ }^{(1)}$. It varies in length from $2-20 \mathrm{~cm}$, the average being about $9 \mathrm{~cm}$. The lifetime risk of developing appendicitis is $8.6 \%$ for males and $6.7 \%$ for females, with the highest incidence in the second and third decades ${ }^{(2-3)}$.

Early reports of perityphlitis and typhlitis in the 19th century appeared to describe clinical features of right sided abdominal pain. Confusion over this right lower quadrant pain existed until Reginald H. Fitz coined the term appendicitis in 1886, and correctly described the appendix as the primary source of inflammation in acute typhlitis ${ }^{(4)}$.

Acute appendicitis is the most common surgical abdominal emergency with a life time prevalence of 1 in 7 individuals ${ }^{(5)}$. The clinical diagnosis remains challenging, because the symptoms are frequently overlapped with Symptoms of other diseases ${ }^{(6)}$.

Despite all improvements in clinical and laboratory methods to diagnose acute appendicitis, the important decisions whether to do appendectomy or not remain challenging (6)

The common surgical practice has been advocated to operate on doubtful cases rather than to wait and see till the diagnosis is certain. This resulted in negative appendectomy rate of around $15 \%$ has been considered acceptable ${ }^{(6-7)}$. The removal of a normal appendix is not a simple procedure and carries a definitive morbidity ${ }^{(7)}$.

In the medical practice, the aim is to diagnose and treat all patients with suspected acute appendicitis without unnecessary appendectomies. There is significant morbidity and mortality associated with acute appendicitis especially when perforation occurred ${ }^{(7)}$. In order to improve the diagnostic accuracy, different aids were introduced like different scoring systems, computer aided programs, Ultrasonography, Computerized tomography, Magnetic resonance imaging, Gastrointestinal contrast studies and laparoscopy ${ }^{(8)}$.

Among these modalities, Ultrasonography is simple, easily available, noninvasive, convenient and cost effective. The ultrasonographic diagnosis of acute 
appendicitis was first introduced by Puylaert in 1986, one hundred years after the publication of first paper on acute appendicitis by Fitz ${ }^{(9)}$.

Over the last 30 years, this technique has been extensively studied and improved. Although the development of US technique has led to dramatic improvements in contrast, spatial and temporal resolution, US examination technique and US signs of appendicitis in real time US have undergone only slight evolution. Graded compression US is performed in a step-wise approach and aims to optimize visualization of the appendix ${ }^{(10,11)}$.

In graded compression technique: where a uniform pressure is applied in RIF by a hand held US transducer, normal and gas filled loops of intestine are either displaced from the field of vision or compressed between anterior and posterior abdominal walls. Inflamed appendix being incompressible and thus optimally seen as a blind ended tubular structure with laminated wall arising from the base of caecum, it is a peristaltic, and its diameter should be more than $6 \mathrm{~mm}{ }^{(12)}$ (figure 1).

Appendicoliths appear as bright echogenic foci with distal acoustic shadowing, and their visualization is another contributory finding. Similarly there may be increased echogenicity of the periappendiceal fat; Ultrasonic probe tenderness can be elicited ${ }^{(13)}$.

Lim HK and Quillin SP had described the usefulness of color Doppler in detecting inflamed appendix. The inflamed thick walled, non-compressible appendix fixed in position by compressing transducer will show circumferential color in contrast to the normal gut which is thin walled and compliant with frequent peristalsis transmitting no or minimum signals. Doppler signals disappear when gangrene or perforation occur ${ }^{(14,15)}$.

A recent study demonstrated that increased use of pre-operative imaging in patients with acute appendicitis resulted in a cost-effective way to decrease the negative appendicectomy rate (NAR) ${ }^{(16)}$.
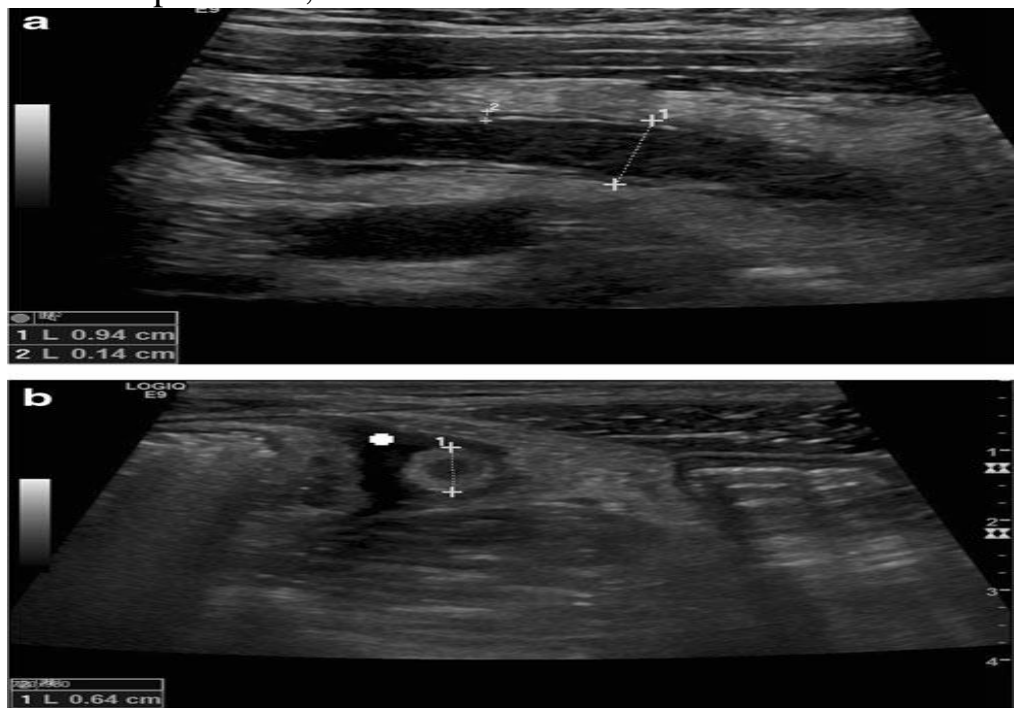

Figure (1) : Longitudinal (a) and transverse (b) real-time US scan of acute appendicitis with thickening of the wall, target-sign, diameter $>6 \mathrm{~mm}$ and free fluid surrounding the appendix

\section{METHODS}

A cross sectional study conducted in AlKindy Teaching Hospital through one year duration between $1 / 5 / 2015$ to $1 / 5 / 2016$.

The inclusion criteria included all patients who are diagnosed and with positive criteria of acute appendicitis .The last sample size was 215 patients. Patients with appendicular mass were excluded from this study.

Data was collected by using a specially designed questionnaire form. After full physical examination, GUE, WBC the patients sent for Ultrasonographic examination .This

examination was done in the outpatient's clinic by the General Electric GE- Volsuen ultrasound machine utilizing 3.5 MHZ convex probe and 7.5 MHZ linear probe.

To detect the vermiform appendix graded compression technique was used. The Ultrasonography findings were recorded as positive and negative for acute appendicitis. 
The criteria for positivity-included (17) visualization of non - compressible tubular and blind-ended non-peristaltic structure with diameter of $6 \mathrm{~mm}$ or more in right lower quadrant, the demonstration of Appendicoliths, Probe tenderness, increased echogenicity of the peri-appendiceal fat and free intraperitoneal fluid particularly in RIF or pelvis. The criteria of negativity ${ }^{(17)}$ were nonvisualization of appendix or visualization of normal appendix with or without alternative diagnosis. Positive and negative appendices on histopathology were regarded in accordance to the following criteria: Negative appendectomy was defined as normal looking appendix and absence of acute inflammation on histopathology, while positive cases included appendices showing acute inflammatory changes.

\section{RESULTS}

A total of 215 patients with suspected acute appendicitis were included in this study with age range from 2-50years (mean age as 23.49 \pm 10.93 years) there was $112(52.09 \%)$ male \&103(47.9\%) female with M: F ratio was (1.08:1), mean age of male patients was $22.51 \pm 9.89$ years and mean age of female patients was $24.56( \pm 11.93)$ years.

Two hindered fifteen cases of suspected acute appendicitis that were included in our study, ultrasounds and histopathlogical examination were done for all cases. Ultrasound results were positive in 175 patients, true positive in 173 patients, and false positive in 2 patients. Ultrasound results were negative in 40 patients' true negative in 13 patients and false negative in 27 patients.

Regarding histopathlogical finding, the results were acutely inflamed appendix 200cases (93.02\%) and normal appendix 15 cases (6.97\%). Statistical analysis showed that graded compression ultrasound yielded a sensitivity $86.5 \%$, specificity $86.6 \%$, diagnostic accuracy $86.5 \%$, positive predictability power of $99.8 \%$ and negative predictability power of $32.5 \%$. All these findings shown in table1.

\section{DISCUSSION}

Even though the diagnosis of acute appendicitis is still thought to be a clinical one, a significant number of patients have normal appendices at surgery (around $15 \%)^{(6,7)}$. Ultrasound has also been shown to be highly sensitive and specific for the diagnosis of not only acute appendicitis but also other conditions that cause right lower quadrant pain (18). The accuracy rate of US in diagnosing acute appendicitis in comparison to histopathology results was $86.5 \%$ with sensitivity and specificity $86.5 \%$ and $86.6 \%$, respectively, which agree with the study done in north of Iraq by Hiwa $\mathrm{O}$, when he mentioned that the accuracy and sensitivity of US was $83.3 \%, 82.1 \%$ but the specifity (100\%) was inconsistent with the present study (19). Moreover these findings are inconsistent with results of other Iraqi study Abdulsalam BM (20) which reported US accuracy of $95.5 \%$, sensitivity $81.8 \%$ and specificity $98 \%$.

In Korea, a large meta-analysis on the role of graded compression US in the diagnosis of acute appendicitis was carried out a few years ago, including 22 articles, the overall sensitivity and specificity were $86.7 \%$ (85.4 $88 \%$ ) and $90.0 \%$ (88.9-91.2), respectively. In particular, their study suggested that US could be useful for the diagnosis of acute appendicitis, especially when patients were younger age, male, and highly clinical suggestive ${ }^{(21)}$, these findings was similar to our result. However our US accuracy in diagnosing acute appendicitis was inconsistent with results of Parsijani et al study in Iran which found US accuracy as $73.6 \%$, sensitivity as $75 \%$ and specificity as $69.2 \%$.

These differences in result of studies might be attributed to differences in sample size, US technique and operators experiences. Positive predictive and negative predictive values of US in present study were $99.8 \%$ and $32.5 \%$ respectively, these results were inconsistent with previous Iraqi study by Abdulsalam BM ${ }^{(20)}$ which showed positive predictive value as $90 \%$, and negative predictive value as $96.2 \%$. This should lead us to the conclusion that when ultrasound report revealed that the appendix was normal, so we should rely on our clinical judgment than the report or perhaps use another modality such as CT scan if possible. There were a significant difference between positive and negative predictive value of sonography confirming our results by Nasiri et al ${ }^{(22)}$ (97.4\% for PPV in comparison to $25 \%$ for NPV) and hiwa $\mathrm{O}^{(19)}$ (100\%for PPV in comparisonto26.6\% for NPV).

Our results emphasize again that a positive ultrasonography for appendicitis is strongly in 
favor of a diagnosis of acute appendicitis. However, a negative ultrasound is not Table (1): Validity of ultrasound diagnosis of acute appendicitis compared with histopathlogical examination as gold standard sufficient to rule out the diagnosis and discharge the patient.

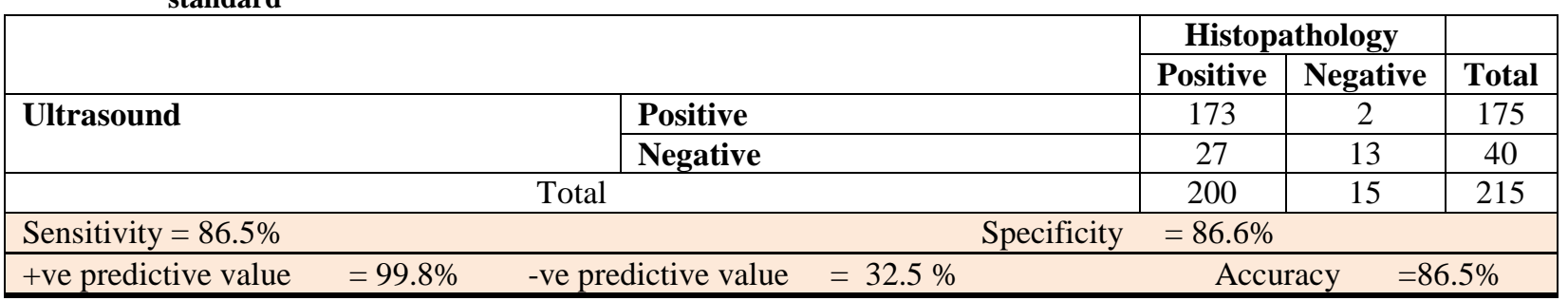

\section{REFRENCES}

1. Dox I, Melloni BJ, Eisner GM (eds) Mellonis illustrated medical dictionary 4th edition, New York: Informa healthcare, 2010.P58

2. William NS,Bulstrod C JK\&O' Connell P R(eds) The vermiform appendix (71) Baily \& Love's short practice of surgery 26th.London New York. Taylor \&Francis Group 2013; p1199-1203

3. Addiss DG, Shaffer N, Fowler BS and Tauxe RV. The epidemiology of appendicitis and appendectomy in the United States. Am $J$ Epidemiol. 1990; 132:910-925

4. Berry JJr, Malt RA. Appendicitis near its centenary .Ann Surg 1984;200:567-75

5. Gwynn LK. The diagnosis of acute appendicitis: clinical assessment versus computed tomography evaluation. J Emerg Med 2001;21:119-123

6. Shogilev DJ, Duus N, Odom SR, Shapiro NI .Diagnosing appendicitis: evidence-based review of the diagnostic approach in 2014. West J Emerg Med. 2014; 15:859-71

7. Humes DJ, Simpson J . Acute appendicitis. BMJ 2006; 333: 530-34

8. Douglas CD, Macpherson NE, Davidson PM, Gani JS. Randomized controlled trial of ultrasonography in diagnosis of acute appendicitis ,incorporating the Alvarado score. BMJ. 2000; 321(7266); 919-22

9. Puylaert JB.Acute appendicitis: US evaluation using graded compression. Radiology. 1986;161:691-95

10. Quigley AJ, Stafrace S . Ultrasound assessment of acute appendicitis in paediatric patients: methodology and pictorial overview of findings seen. Insights Imaging. 2013;4(6):741-51

11. Birnbaum BA, Wilson SR .Appendicitis in the millennium. Rad.2000; 215(2):337-48 